\title{
Absence of single critical dose for the amorphization of quartz under ion irradiation
}

\section{Zhang, Shuo}

2018-01-10

Zhang , S , Pakarinen , O H , Backholm , M , Djurabekova , F, Nordlund , K, Keinonen , J \& Wang , T S 2018 , ' Absence of single critical dose for the amorphization of quartz under ion irradiation ' , Journal of Physics. Condensed Matter , vol. 30 , no. 1 , 015403 . https://doi.org/10.1088/1361-648X/aa9

http://hdl.handle.net/10138/308627

https://doi.org/10.1088/1361-648X/aa9868

acceptedVersion

Downloaded from Helda, University of Helsinki institutional repository.

This is an electronic reprint of the original article.

This reprint may differ from the original in pagination and typographic detail.

Please cite the original version. 


\title{
Absence of single critical dose for the amorphization of quartz under ion irradiation
}

\author{
S, Zhang (张硕) \\ School of Nuclear Science and Technology, Lanzhou University, \\ Lanzhou, Gansu Province 730000, People's Republic of China and \\ Department of Physics, P. O. Box 43, FIN-00014 University of Helsinki, Finland \\ O. H. Pakarinen, M. Backholm, F. Djurabekova, K. Nordlund, J. Keinonen \\ Helsinki Institute of Physics and Department of Physics, \\ P. O. Box 43, FIN-00014 University of Helsinki, Finland
}

\section{T.S. Wang*}

School of Nuclear Science and Technology, Lanzhou University, Lanzhou, Gansu Province 730000, People's Republic of China

(Dated: December 28, 2017)

In this work, we first simulated the amorphization of crystalline quartz under $50 \mathrm{keV}{ }^{23} \mathrm{Na}$ ion irradiation with classical molecular dynamics (MD). We then used binary collision approximation algorithms to simulate the Rutherford backscattering spectrometry in channeling conditions (RBS-C) from these irradiated MD cells, and compared the RBS-C spectra with experiments. The simulated RBS-C results show an agreement with experiments in the evolution of amorphization as a function of dose, showing what appears to be (by this measure) full amorphization at about $2.2 \mathrm{eV} /$ atom. We also applied other analysis methods, such as angular structure factor, Wigner-Seitz, coordination analysis and topological analysis, to analyze the structural evolution of the irradiated MD cells. The results show that the atomic-level structure of the sample keeps evolving after the RBS signal has saturated, until the dose of about $5 \mathrm{eV}$ /atom. The continued evolution of the $\mathrm{SiO}_{2}$ structure makes the definition of what is, on the atomic level, an amorphized quartz ambiguous.

PACS numbers: $64.70 . \mathrm{kp}, 61.43 . \mathrm{Dq}, 61.72 . \mathrm{Cc}, 61.82 . \mathrm{Fk}$

* corresponding author: tswang@lzu.edu.cn 


\section{INTRODUCTION}

Most semiconductors and many insulators amorphize during energetic ion or neutron bombardment. The mechanisms causing the amorphization have been extensively studied, and a fairly good understanding has been obtained [1-15]. However, multiple experimental and simulation studies show that the amorphization process can be quite complex, involving several different stages [13] and mechanisms [16]. Moreover, different experimental analysis methods can give different levels of amorphization even for exactly the same material and irradiation [6, 17, 18]. To obtain an atom-level understanding of the process underlying the amorphization, computer simulation techniques, such as molecular dynamics (MD) [19], have proven to be very useful [9, 10, 12-14, 20]. Previous atom-level studies of amorphization have, however, focused on pure elements or compounds with high-symmetry crystal structures.

$\mathrm{SiO}_{2}$ is a material of great interest due to its wide use as an insulator in Si microelectronics [21] and as one of the base materials for present and future optoelectronics [22]. Its crystalline equilibrium state, $\alpha$-quartz, can be rendered amorphous by ion irradiation [23-28]. The atom-level mechanisms behind this amorphization are not, however, well understood. Previous studies of the irradiation response of $\mathrm{SiO}_{2}$ to amorphization have given valuable insights on defect production by single recoils in the $\beta$-cristobalite structure $[29,30]$, but simulation of full amorphization process starting from $\alpha$-quartz has never been performed. In the current paper we use atomistic simulations to examine the amorphization of quartz, with the particular aim to understand how different atomic-level measures of the degree of amorphization compare with each other.

\section{METHODS}

\section{A. Amorphization simulation}

In the present work, we perform the classical molecular dynamic (MD) simulations of complete amorphization process of $\alpha$-quartz. Although, in previous experiments [23-25], there were a number of different ions, such as, $\mathrm{B}^{+}$, $\mathrm{Ar}^{+}, \mathrm{H}^{+}, \mathrm{He}^{+}$and $\mathrm{Na}^{+}$etc., used to amorphize $\alpha$-quartz by ion irradiation, we selected for concreteness the $50 \mathrm{keV}$ ${ }^{23} \mathrm{Na}$ ions to study the amorphization process of quartz on atomistic level. The simulations were carried out by the following two steps.

In the first step, an energetic recoil with a random direction was used to initiate a cascade at about the center of the simulation box, which was started from a perfect crystalline $\alpha$-quartz structure and relaxed at $77 \mathrm{~K}$ temperature (the same temperature as in Ref. 23). The evolution of the cascade was followed for 20 ps and the border of the cell was cooled down using Berendsen temperature control method towards $77 \mathrm{~K}$ to remove the additional energy introduced by recoils. The electronic energy loss of the energetic atoms with energy higher than 10 eV was taken into account as a frictional force during their movement. The high energy of the recoils prevents the simulations from using a large time-step, therefore, an variable time-step with a maximum displacement $0.05 \AA$ per time-step was used to ensure the efficiency of the simulations. The additional momentum introduced by the recoils would lead to a collective movement of atoms in the cells (i.e. a velocity of the center of mass). To ensure the accuracy of the temperature in the MD simulations, the velocity of the center of mass was removed every time-step.

In the second step, the pressure in the cell was relaxed to zero and the temperature was cooled back to the starting temperature of $77 \mathrm{~K}$ in the whole cell during the following $5 \mathrm{ps}$. Then, the cell was shifted randomly over the periodic boundary conditions to obtain a random distribution of the starting positions for the next recoils [16]. These two steps were iterated until the cell was fully amorphized. The final structure resembles the amorphous silica, however, the properties of irradiation-induced amorphous silica structure may not be the same as vitreous silica glasses [31].

These simulations were carried out by the MD code LAMMPS [32] and the Vashishta interatomic potential [33] was used for the simulations. A cubic cell with size roughly $5 \times 5 \times 5 \mathrm{~nm}^{3}$ containing about 10000 atoms was chosen to ensure it is large enough to keep the cascade inside the boundaries of the cell. Periodic boundary conditions were used in all directions of the simulation cells.

The effect of recoil energy distribution is studied in two ways: with four series of simulations with mono-energetic recoils of $30,100,300$ and $500 \mathrm{eV}$, respectively, and with one series of simulation with a realistic recoil spectrum calculated for a $50 \mathrm{keV}{ }^{23} \mathrm{Na}$ ion, matching typical amorphization experiments in quartz [23]. The latter one is also important for checking if low-energy recoils are causing a self-healing effect to the existing damage, as observed for amorphization of silicon [12]. The recoil spectrum was calculated with the range calculation code MDRANGE $[34,35]$, which takes the many-body interactions between ions and atoms into account to simulate the slowing down of energetic ions inside materials. The recoil energy spectrum is limited to $3-1000$ eV to decrease the simulation time needed to compute negligibly low energy recoils, and on the other hand to limit the system size needed for very rare high energy recoils. 


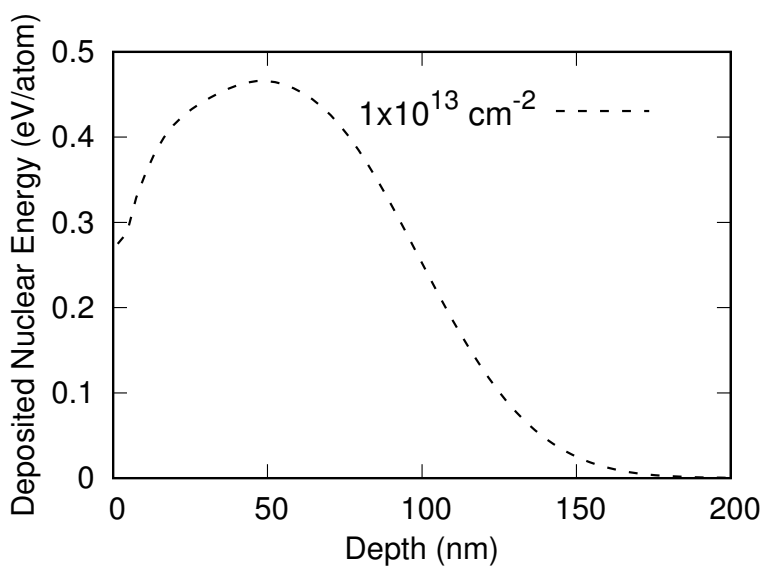

FIG. 1. Depth profile of deposited nuclear energy of $50 \mathrm{keV}^{23} \mathrm{Na}$ at fluence $1 \times 10^{13} \mathrm{~cm}^{-2}$ in quartz. Here the deposited energies were calculated by adding the energies of primary recoils in certain depth together.

The mono-energetic recoil simulations showed very similar behavior to those using the realistic recoil spectrum, and also the differences between single recoil energies was very small. Therefore, in the remainder of this paper, we discuss the result using recoil spectrum unless otherwise mentioned. To enable comparison with experimental fluences, we translated the recoil energies to fluence as follows. The MD simulations naturally give the dose in $\mathrm{eV} / \mathrm{atom}$ for each recoil event by taking the recoil energy minus the energy lost to electronic stopping, and dividing this with the number of atoms. This was then translated from the nuclear energy deposition curve (Fig. 1) into a fluence, using the maximum energy deposition depth as the reference point.

\section{B. Analysis methods}

In order to assess the degree of amorphousness and the structural evolution of irradiated simulation $\alpha$-quartz cells, we employed several different approaches to analyze the MD simulation results.

Rutherford Backscattering Spectrometry in channeling conditions (RBS-C) is a very powerful method to measure the amorphization state of a crystalline structure $[1,4,5,13,28,36,37]$. To directly compare with the experimental RBS-C spectra, we use the recently developed simulation code Rutherford Backscattering Simulation in Arbitrary DEfective Crystals (RBSADEC) [38] to simulate the RBS-C spectra from these MD cells. This code, based on the binary collision approximation (BCA) algorithm, allows us to simulate RBS-C spectra from an damaged crystal described by a set of atom coordinates in a simulation cell with a size comparable to the experimental scale in the $z$ dimension.

The RBS-C simulation procedure was the same as that used in Ref. 39 for metal alloys, and summarized in the following. First we used the software Stopping and Range of Ions in Matter (SRIM) [40] calculating the depth profiles of deposited nuclear energy of $50 \mathrm{keV} \mathrm{Na}+$ ions in quartz (see fig. 1). The deposited nuclear energy at every depth is obtained by adding up all the energy of primary recoils at this depth together. Then, for a certain irradiation fluence, the MD cells are merged along the depth direction in such an order that the doses to what the given cell was irradiated corresponded to the energy deposited at the respective depth. Finally, the RBS-C spectra from the merged cells (which are referred to as BCA cells in the remainder of this paper) are simulated by RBSADEC. In these RBS-C simulations, the energy of probe $\alpha$ ions is same with the experiment in Ref. $23,900 \mathrm{keV}$, and the incident angle was allowed to have an uncertainty range from $0.1^{\circ}$ to $0.4^{\circ}$ to emulate the effect of various systematic uncertainties in the experiment. The backscattered signals are collected at $160^{\circ}$ of the incident direction.

We also employed the angular structure factor analysis approach [41, 42], which measures the degree of disorder of an atom based on its local environment, and hence can give an atomic-level insight into evolution of amorphization processes. In this approach, a list of all angles between bonds from an atom to its neighbors is formed. The comparison of these angles to those in the perfect crystalline structure gives an angular structure factor $P_{s t}(i)$ of each atom, which 
can be written as,

$$
\begin{gathered}
P_{s t}=\frac{1}{P_{u}(i)}\left(\sum_{j}\left[\theta_{i}(j)-\theta_{i}^{p}(j)\right]^{2}\right)^{1 / 2} \\
P_{u}=\left(\sum_{j}\left[\theta_{i}^{u}(j)-\theta_{i}^{p}(j)\right]^{2}\right)^{1 / 2}
\end{gathered}
$$

where $\theta_{i}(j)$ are the angles formed between atom $\mathrm{i}$ and its neighbors, $\theta_{i}^{p}(j)$ is the distribution of angles in a perfect lattice, $\theta_{i}^{u}(j)=j \pi / n(n-1) / 2$ is the uniform angular distribution and $n$ is the number of neighbor atoms. The $P_{s t}$ values can be calibrated against the distribution of values in a fully crystalline and amorphous reference cell. The latter is obtained from cells made by repetitive annealing in Ref. 43 . For $\mathrm{SiO}_{2}$, neighbors up to a distance of $3.8 \AA$ are included in the analysis, and an atom is interpreted to be part of amorphous neighborhood if both the atom and most of its neighbor have a structure factor higher than 0.2 . This approach is found to detect exactly 0 amorphous atoms in a 10800 atom quartz simulation cell at $77 \mathrm{~K}$, and more than $97 \%$ of atoms in the reference amorphous cell as amorphous atoms. In the present work, the fraction of atoms that are labeled amorphous is used as the degree of amorphization of structures.

The Wigner-Seitz analysis method implemented by OVITO [44] was also used to count the number of defects in the simulation MD cells. Since the volume of the MD cells would increase with the irradiation doses, in the present analysis the size of the simulation boxes were scaled to unity to ensure the accuracy of the volume of Wigner-Seitz cells. The obtained number of Wigner-Seitz defects was used to measure the degree of amorphousness of the cells.

For the selected doses of $0,0.5,2.0,3.0$ and $7.0 \mathrm{eV} /$ atom, with 0 representing the beginning of irradiation process and 7.0 its final state after the amorphization has been reached, we analyze the radial distribution function for all the atoms in each cell. The first peak in the function gave us a cutoff distance $r_{\text {cut }}=2.0 \AA$, taken at the maximum of the nearest-neighbor in the radial distribution analysis (cf. section III C) to account the coordination defects formed in the cell during the simulation of irradiation process.

For coordination defects we count the ill-coordinated atoms with the lack or excess of bonds compared to those in a perfect structure. Recall that in perfect quartz structure all Si atoms have 4 nearest-neighbor bonds to $\mathrm{O}$, and all $\mathrm{O}$ atoms 2 nearest-neighbor bonds to Si. Thus Si atoms with less or more than 4 neighbors are interpreted to be underand over-coordinated, respectively.

Quartz is composed of $\mathrm{Si}-4 \mathrm{O}$ tetrahedra, which shares each of their four vertices with neighboring tetrahedra. Such a clearly defined structure can be characterized by circuit connections (rings) of Si-O bonds. Analysis of topological evolution of the rings during the irradiation process may provide a valuable insight in the process of amorphization of quartz [45-49]. In the present work, we use the code RINGS [50] to analyze the primitive ring-structure changes during the amorphization process induced by ion irradiation.

\section{RESULTS}

\section{A. Comparison with RBS experiments}

To directly compare with the experiments by Harbsmeier and Bolse [23], we simulated the RBS-C spectra from the BCA cells irradiated at fluences $2 \times 10^{13}, 3 \times 10^{13}, 4 \times 10^{13}$ and $5 \times 10^{13} \mathrm{~cm}^{-2}$. The comparison of the experimental RBS-C spectra and the simulated ones are shown in Fig. 2. The experimental spectra with fluence of $2 \times 10^{13}, 3 \times 10^{13}$, $4 \times 10^{13}$ and $5 \times 10^{13} \mathrm{~cm}^{-2}$ were denoted by the green, cyan, blue and red markers, respectively, while the simulated spectra are shown by the solid line with corresponding colors.

The simulated spectra can be considered to agree well with the experimental results, considering the following factors: (i) no fitting parameters are used for these simulations and both the MD and BCA simulations are carried out independently; (ii) the amorphization process induced by ion-irradiation is quite complex and related to various properties of $\alpha$-quartz, the semi-empirical atomic interaction potential used in these MD simulations could not fit all the related properties well; (iii) the size of the MD simulation cell is much smaller than experimental samples, which prevents the formation of defect clusters with the size larger than the simulation cells; (iv) the irradiation dose rate in the MD is much higher than in experiments, making possible that defect migration or thermal recombination is underestimated. This issue is discussed further in Section IV A and (v) interactions of low-energy ions and matters are quite complex [51, 52] and MD simulations could not take all the details into account. The discrepancies between the simulated and experimental spectra are mainly reflected in two aspects: (i) Except for the spectrum obtained for the high irradiation fluence of $5 \times 10^{13} \mathrm{~cm}^{-2}$, which is rather similar to the spectrum of the random structure, the simulated spectra show broader damage peaks than the experiments. This indicates insufficient relaxation of 


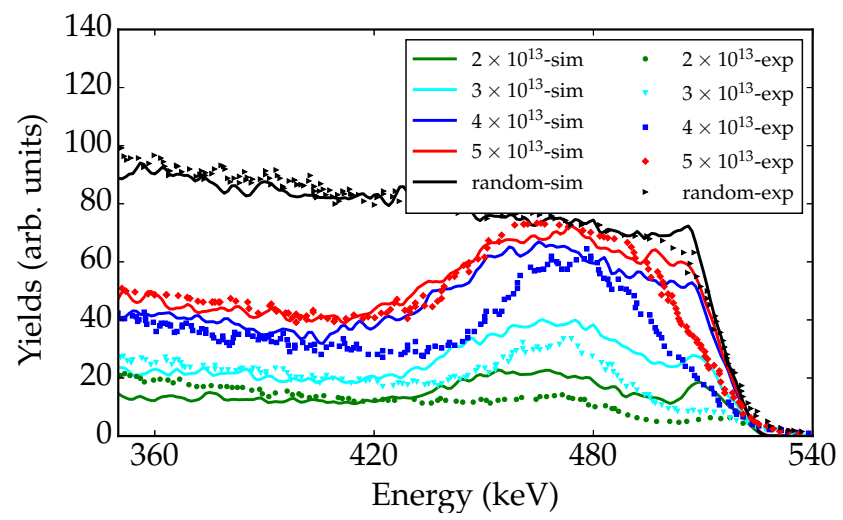

FIG. 2. Comparison of simulated RBS/C spectra shown by solid lines and experimental results shown by markers.

the structure between ion impacts in the high irradiation fluence MD simulations, compared to the experimental irradiation condition. (ii) The simulated spectra show that the RBS yield profiles start at the surface (energy range from $\sim 500$ to $\sim 520 \mathrm{keV}$, see Fig. 2) as the deposited energy shown in Fig. 1, while the experimental ones show obviously less damage in this region. This indicates that in the experiments, the surface is an effective sink for irradiation induced damage.

Moreover, we note that the difference between experiments and simulations is larger for low irradiation fluence $\left(2 \times 10^{13}\right.$ and $\left.3 \times 10^{13} \mathrm{~cm}^{-2}\right)$ than that for high fluence $\left(4 \times 10^{13}\right.$ and $5 \times 10^{13} \mathrm{~cm}^{-2}$, Fig. 2$)$ This is likely due to ion channeling effects [53]. The channeling effect of irradiating ions $\left(\mathrm{Na}^{+}\right)$in crystalline quartz would lead to less primary recoils than the predictions of SRIM simulations. In the quartz samples irradiated at low fluence, more irradiating ions get into samples in channeling conditions. Thus, the depth profiles of deposited nuclear energy calculated by SRIM (Fig. 1) is inaccurate for the low irradiation fluence case. That is one of the likely reasons for the larger difference at low irradiation fluence.

One more important issue with respect to the comparison of experimental and simulation RBS-C spectra is worth mentioning here. H. Fischer et al. reported that $1.4 \mathrm{MeV} \mathrm{He}$ ions irradiation at fluence of $\sim 10^{16} \mathrm{~cm}^{-2} \mathrm{could}^{-4}$ substantially increase the damage produced in quartz by $150 \mathrm{keV}$ Ar ions [25]. In the present simulations and the experiment in the Ref. 23, the probe ions (900 keV He ions) used in the RBS-C measurements may in principle also increase the damage during the RBS-C measurement. However, we did not take this effect into account, because the implantation fluence of RBS-C probe ions was (and should be) low enough that the damage induced by the probe ions could be neglected. According to our RBS-C simulations, getting a RBS-C spectra with good statistical properties in experiments only needs a implantation fluence much lower than $10^{16} \mathrm{~cm}^{-2}$, which is the fluence reported by Fisher et al. for the visible damage induced by He ions.

The damage peaks in the RBS energy spectra (Fig. 2) are located at $\sim 470 \mathrm{keV}$. Following common practise in experiments, we extracted the maximum RBS yields $\left(\chi_{\max }(\phi)\right)$ of these damage peaks from the spectra with different irradiation fluences $(\phi)$, and normalized them by the yields at $470 \mathrm{keV}$ of the random spectrum. The normalized $\chi_{\max }(\phi)$ can be used to denote the degree of the damage. Comparison of $\chi_{\max }(\phi)$ obtained in our simulations and in the experiments [23] is shown in Fig. 3.

Evolution of this parameter, $\chi_{\max }(\phi)$, with irradiation fluence is related to the damage accumulation processes of the irradiation. Thus, we use 3 models to fit our RBS simulation data:

- Direct impact model implies that amorphization can be achieved locally in a single collision cascade [54]. For this model, the saturation-like behavior is expected to:

$$
\chi(\phi)=1-\exp (-\sigma \phi)
$$

- Gibbons overlap model assumes that a single cascade generate only some defects and the amorphization occurs ion-induced defects overlap [55]:

$$
\chi(\phi)=1-\sum_{k=0}^{n-1} \frac{(\sigma \phi)^{k}}{k !} \exp (-\sigma \phi)
$$

Here we consider two cases of this model with $n=2$, Gibbons single overlap model,

$$
\chi(\phi)=1-(1+\sigma \phi) \exp (-\sigma \phi)
$$




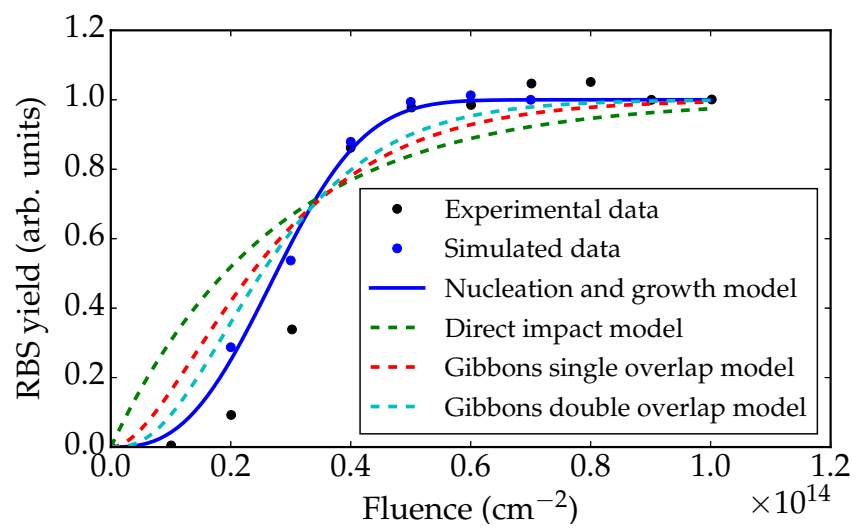

FIG. 3. Comparison of the maximum damage obtained by the present RBS-C simulations (blue markers) and experimental results [23] (black markers) and fits to the simulated RBS data by the Direct impact model (green dashed line), Gibbons single overlap model (red dashed line), Gibbons double overlap model (cyan dashed line) and nucleation and growth model (blue solid line). The fits by the nucleation and growth model with exponent parameter $n=2.74$ describes the simulated data best.

and for $n=3$, Gibbons double overlap model,

$$
\chi(\phi)=1-\left(1+\sigma \phi+\frac{1}{2} \sigma^{2} \phi^{2}\right) \exp (-\sigma \phi)
$$

- Nucleation and growth model implies that the defects generated by collision cascades migrate, nucleate and grow $[56,57]$ :

$$
\chi(\phi)=1-\exp \left(-(\sigma \phi)^{n}\right)
$$

As shown in Fig. 3, the nucleation and growth model describes the simulated values of degree of damage, $\chi_{\max }(\phi)$ the best. It also gives a very good agreement with the experiment data, where the critical fluence for full amorphization is about $5 \times 10^{13} \mathrm{Na} / \mathrm{cm}^{2}$ (about $2.2 \mathrm{eV} /$ atom). Here the corresponding irradiation dose could be obtained by multiplying the maximum deposited energy value $F_{D}^{\max }=4.5 \times 10^{-14} \frac{\mathrm{eV} \cdot \mathrm{cm}^{2}}{a t \cdot i o n}$ (see Fig. 1). We obtained a fitting exponent $n=2.74$, which indicates three-dimensional process of defect nucleation and growth. Compared with the nucleation and growth model, neither the Gibbons overlap models nor the direct impact model are able to describe the damage accumulation processes reasonably well.

\section{B. Angular structure factor and Wigner-Seitz analysis}

As another measurement of the degree of damage, the angular structure factors of the irradiated cells with different doses are shown in Fig. 4. The analysis results show that a total irradiation dose of $\sim 3 \mathrm{eV} /$ atom (i.e. $7 \times 10^{13}$ $\mathrm{Na} / \mathrm{cm}^{2}$ ) is needed to make the $\alpha$-quartz fully amorphous. This value is clearly not the same as that obtained from the RBS-C simulations, where it appears that an irradiation dose of $\sim 2.2 \mathrm{eV} /$ atom (i.e. $5 \times 10^{13} \mathrm{Na} / \mathrm{cm}^{2}$ ) could cause fully amorphization of quartz. The difference is not only reflected in the critical dose for full amorphization of quartz, but also the processes of damage accumulation. This can be seen from the fits of angular structure factors by the three models (Fig. 4). As seen from the fits by the three models, both the Gibbons double overlap model and the nucleation and growth model could fit the simulation data well. However, even though the nucleation and growth model could still fit the data well, the exponent parameter $n=1.83$ fitting the angular structure factor does not agree with the fitting result from the RBS data. That indicates the behaviors of damage accumulation derived from these two analysis methods are clearly different.

The number of Wigner-Seitz defects was also used to measure the degree of the damage in the MD cells. The analyzed results were normalized by the defect number at dose of $7.0 \mathrm{eV} /$ atom and show in Fig. 5. Based on the Wigner-Seitz analysis shown by Fig. 5, the critical dose for the amorphization of quartz is about $\sim 4.5 \mathrm{eV} /$ atom. This value is larger than the result obtained by either the RBS or angular structure factor analysis.

Fig. 5 shows that the nucleation and growth model fits the analysis results best, again. A fitting exponent parameter $n=2.24$, which indicates two-dimensional nucleation and growth, was obtained. 


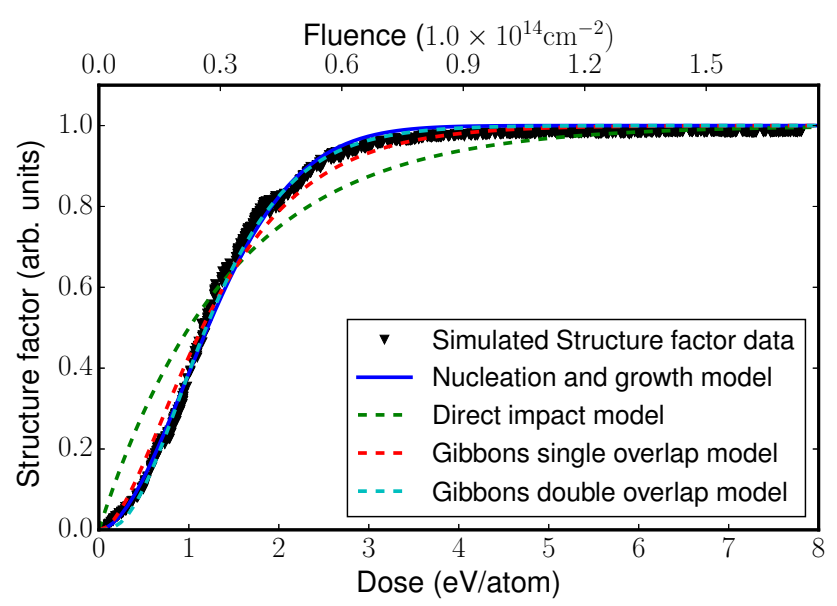

FIG. 4. The angular structure factors of the MD simulated structures with different doses (bottom $x$ axis) or fluence (top $x$ axis) and the fits by the three models (i.e. Direct impact model, Gibbons single, double overlap model and nucleation and growth model).

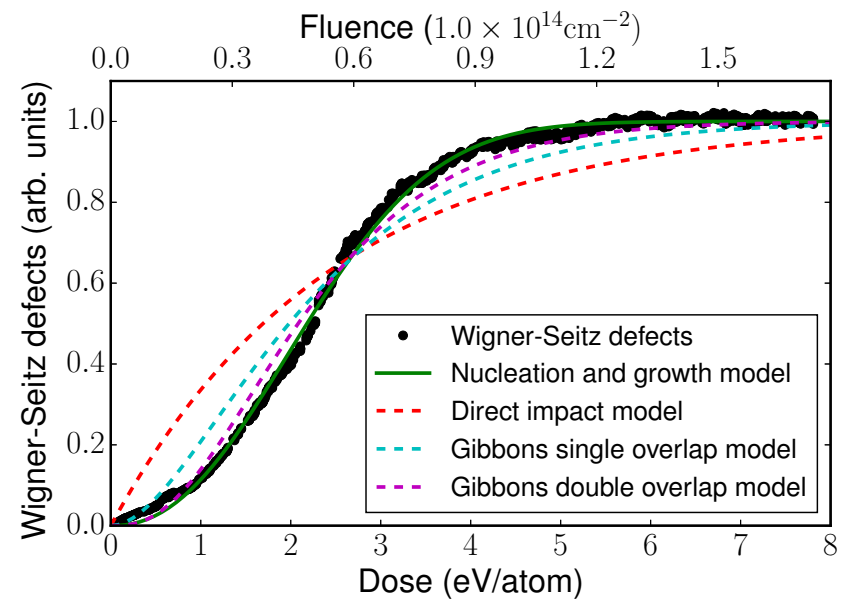

FIG. 5. The number of Wigner-Seitz defects in the MD cells irradiated by different doses (bottom $x$ axis) or fluence (top $x$ axis). Here the defect numbers in every cells were normalized by that in the cell irradiated at dose $7.0 \mathrm{eV} /$ atom, where the total number of atom is 10800 and defect (vacancies and interstitials) number is $\sim 8600$.

\section{Radial distribution function and coordinate analysis}

In Fig. 6, we plot the radial distribution functions at the different irradiation doses $0.5,2.0,3.0$ and $7.0 \mathrm{eV} /$ atom. For reference, we added the same function of the unirradiated cell at $77 \mathrm{~K}$. The radial distribution functions (Fig. 6), show that the two first peaks are well pronounced for all the analyzed structures. That indicates the presence of shortrange order in all irradiated structures. The 3rd and 4th peak can be clearly seen in the unirradiated cell. The 4th peak becomes less distinguishable with the increase of irradiation doses and disappears at the dose to $2 \mathrm{eV} / \mathrm{atom}$. This indicates the absence of long-range order already at this dose, confirming that the structure is rendered amorphous based on this analysis method.

The radial distribution function analysis also showed that in all cases, the nearest-neighbor peak is located at 1.6 $\AA$ and essentially ends at $2.0 \AA$. Thus $2.0 \AA$ was used as a cutoff parameter for the coordination defect analysis. In the coordination analysis, the atoms that have less/more coordination atoms than those in perfect quartz where $\mathrm{Si}$ atoms have 4 neatest-neighbor bonds and $\mathrm{O}$ atoms have 2 nearest-neighbor bonds were regard as under-coordinated/overcoordinated defects. The results show that the under-coordinated defects are dominant in all the irradiated structures. The numbers of under-coordinated atoms in the structures irradiated by different dose are shown in the table I. 


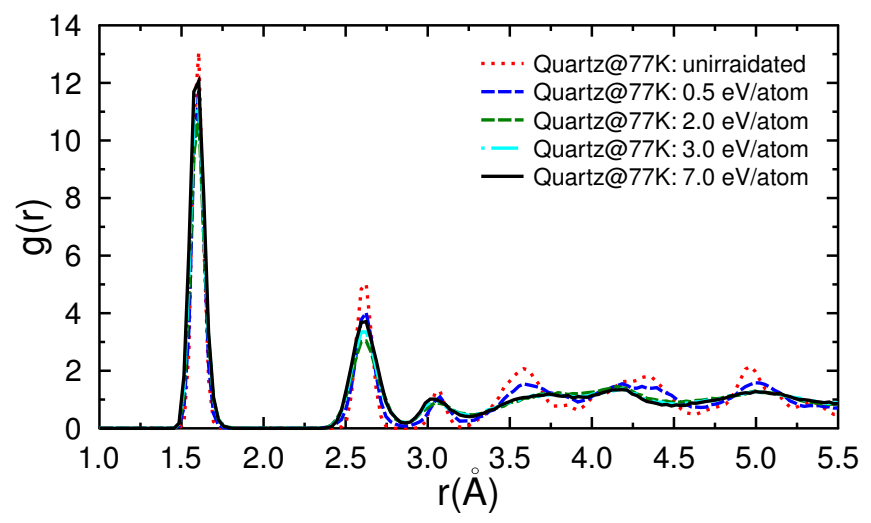

FIG. 6. Radial distribution functions at different doses.

TABLE I. Coordination analysis in irradiated simulation cells for the doses of $0,0.5,2.0,3.0,5.0$ and $7.0 \mathrm{eV} /$ atom. $N_{a t}$ gives the total number of atoms in the simulation cell. The $0 \mathrm{eV} /$ atom case is the initial perfect quartz relaxed at $77 \mathrm{~K}$. The abbreviations "underc." is the under-coordinated atoms compared to perfect quartz where all Si atoms have 4 nearest-neighbor bonds to $\mathrm{O}$ and $\mathrm{O}$ atoms have 2 nearest-neighbor bonds, respectively. The cutoff used in this analysis is $2.0 \AA$.

\begin{tabular}{lccc}
\hline \hline Case & $N_{a t}$ & $\begin{array}{c}\mathrm{Si} \\
\text { underc. } \\
(\%)\end{array}$ & $\begin{array}{c}\mathrm{O} \\
\text { underc. } \\
(\%)\end{array}$ \\
\hline $0 \mathrm{eV} /$ at & 10800 & 0 & 0 \\
$0.5 \mathrm{eV} /$ at & 10800 & 6.0 & 3.06 \\
$2.0 \mathrm{eV} /$ at & 10800 & 12.22 & 6.25 \\
$3.0 \mathrm{eV} /$ at & 10800 & 15.22 & 7.67 \\
$5.0 \mathrm{eV} /$ at & 10800 & 16.86 & 8.52 \\
$7.0 \mathrm{eV} /$ at & 10800 & 16.69 & 8.46 \\
\hline \hline
\end{tabular}

Fig. 7 shows the total energy evolution of the MD simulation cell (solid blue line, right $y$ axis) and the density of irradiated quartz (solid red line, left $y$ axis). The energy of the structure increases rapidly at the beginning of irradiation process and reaches the saturation at the dose of $\sim 3 \mathrm{eV} /$ atom, while the density of the irradiated quartz keeps decreasing until the dose of $\sim 6 \mathrm{eV} /$ atom. The reduction of density is up to $\sim 25 \%$, which does not perfectly fit the experimental observation $\sim 14 \%$ volume expansion [58] and $\sim 19 \%$ volume expansion [23] after ion irradiation. This small difference may be related to the large amount of under-coordinated silicon and oxygen atoms, which we observe in the simulations. Some densification may occur due to partial saturation of the dangling bonds of these atoms between the subsequent cascades, during the time intervals, which are much longer in experiments than in the classical MD. The difference between the density of simulated and experimental amorphous $\mathrm{SiO}_{2}$ may also be related to details of the amorphization conditions. As a point of comparison on another covalent amorphous material, recent work shows that there are significant differences in the structure and density of amorphous Si synthesized by different experimental approaches [59]. We note here that when the value of the potential energy reaches its saturated value at the irradiation dose of about $3 \mathrm{eV} /$ atom, the density of the amorphized quartz at this point was about $2.2 \mathrm{~g} / \mathrm{cm}^{3}$, which is close to the density of vitreous silica. Besides, our simulations show that the density of quartz may continue decreasing with increasing irradiation dose even after the quartz is considered fully amorphized by other methods.

\section{Ring analysis}

Fig. 8(a) and (b) show the ring analysis results of the irradiated MD simulation cells. The red dotted, blue dashed, green dashed and black solid lines in Fig. 8(a) show the ring size distribution of quartz irradiated to doses of 0.5, 1.0, 1.5 and $2.0 \mathrm{eV} /$ atom, respectively. The purple dashed, orange dashed and black lines in figure 8(b) shows the ring size distribution of quartz irradiated at 3,5 and $7 \mathrm{eV} /$ atom. For the un-irradiated quartz, the ring analysis show that only 6-rings (6 Si-O atom bonds) and 8-rings exist, and the average ring size is 7.67. From the figure, we can clearly observe that the total number of rings (sum of rings with any size) decrease significantly with the irradiation dose. 


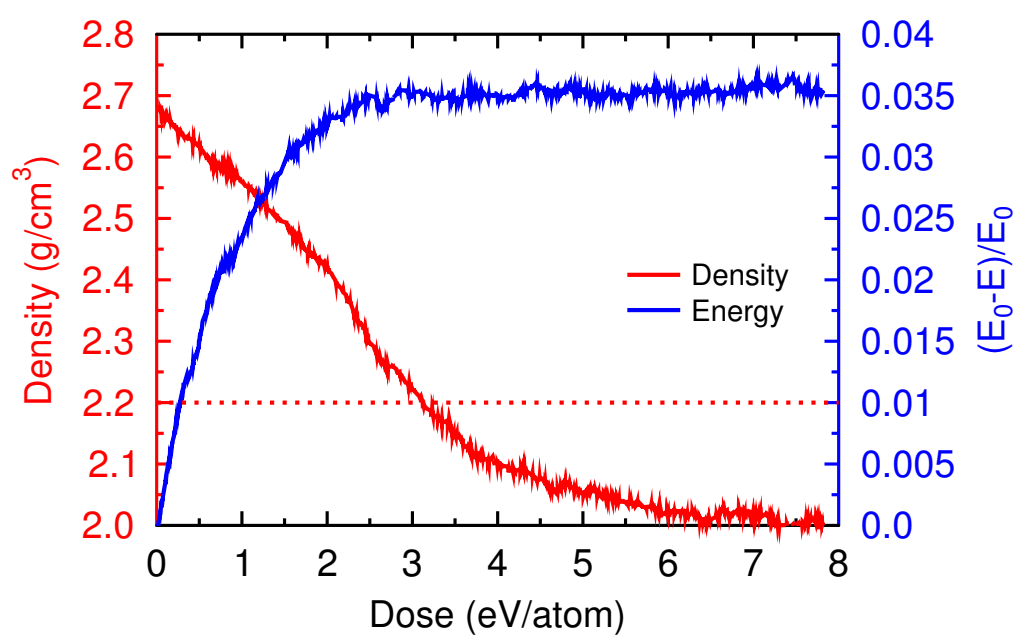

FIG. 7. The energy (solid blue line, right $y$ axis) and density (solid red line, left $y$ axis) evolution during the irradiation. The red dashed line (with left $y$ axis) is a guide line showing the density of vitreous silica, which is about $2.2 \mathrm{~g} / \mathrm{cm}^{-3}$.
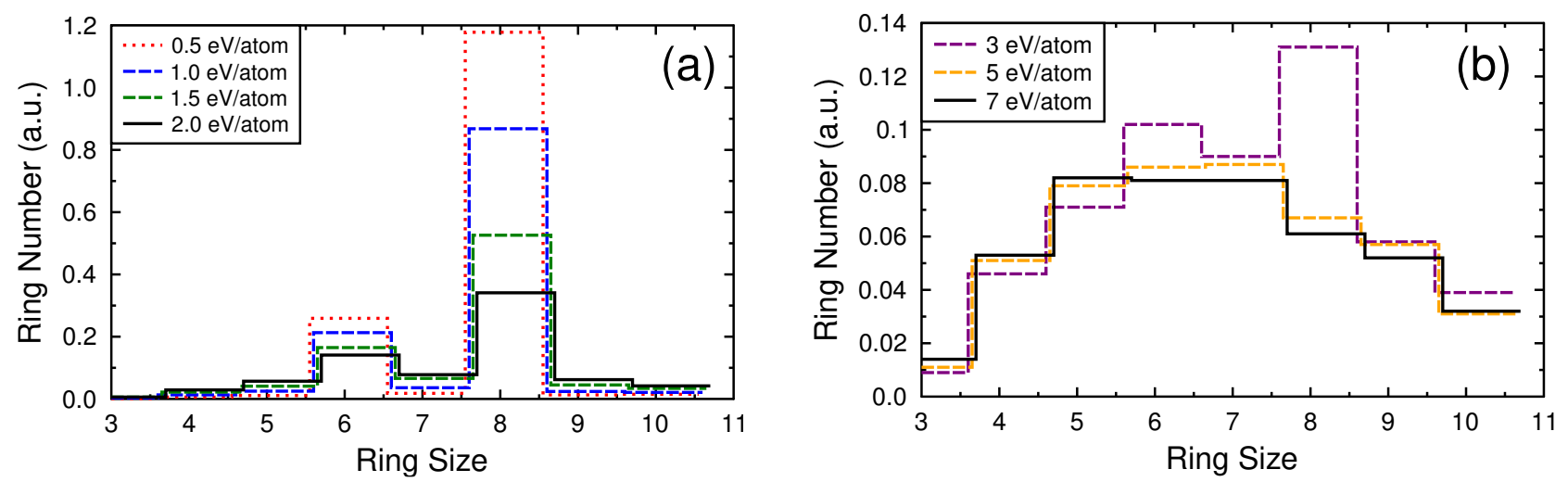

FIG. 8. Histogram of evolution of primitive ring size distribution as the irradiation dose (a) $0.5-2.0 \mathrm{eV} / \mathrm{atom}$; (b) 3.0-7.0 $\mathrm{eV} /$ atom. The number of rings was normalized by the total number of atoms in the simulation cells.

This indicates that quartz shows lower connectivity after the ion irradiation. The lower connectivity of the structure is corresponding to the lower density of the irradiated quartz. As seen from figures 8(a) and 8(b), as the irradiation dose increases, the ring sizes in quartz are no longer dominated by 8-rings, and the distribution of ring sizes is towards a more equitable distribution (see $7 \mathrm{eV} /$ atom in figure $8 \mathrm{~b}$ ). That is consistent with Hobbs and co-workers' work $[45,47]$, who also suggested that the predominant 8-rings in the quartz should decrease during the amorphization of quartz induced by irradiation. For the lower doses (0-5 eV/atom), the number of 8-rings decreases rapidly with dose, however after $5 \mathrm{eV} /$ atom, the ring size distribution does not change significantly. That indicates the ring structure changes nearly reaches an equilibrium state after about $5 \mathrm{eV} /$ atom.

\section{DISCUSSION}

\section{A. Possible effects contributing to amorphization}

By analyzing the details of the calculation, we are able to assess the significance of various effects on the rate of amorphization. Here we consider three possible effects:

1) Long timescale annealing

2) Low-energy recoils

3) Analysis method dependence

The agreement between experimental and simulated RBS-C spectra (Fig. 2) tells us that the MD simulation catches 


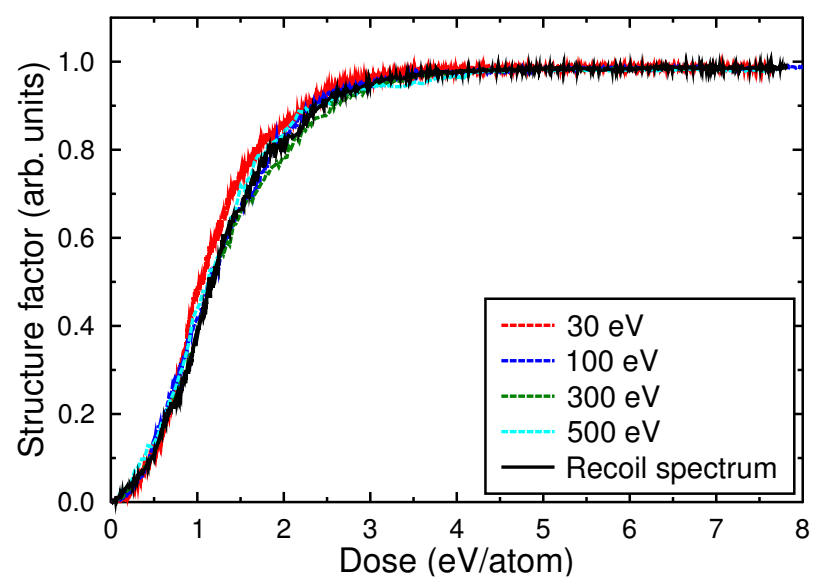

FIG. 9. Comparison of amorphization results obtained with mono-energetic recoils and the recoil spectrum corresponding to $50 \mathrm{keV} \mathrm{Na}$ irradiation.

most of significant details of the amorphization process. However, it is worth mentioning that the MD simulation timescale is much shorter than that in the real experiments. In the MD simulations, every collision cascade was followed until it cooled down to the ambient temperature $(\sim 25 \mathrm{ps})$, while in the experiment, the system has much longer time to relax at the ambient temperature condition ( $77 \mathrm{~K}$ for this case). Even though the long timescale annealing effect was underestimated in the simulations, the agreement between the simulated and experimental RBS-C spectra prove that the long timescale migration and recombination of defects, which could repair damaged structures on longer timescales than those included in the MD simulation, would not significantly affect the process of amorphization of quartz induced by ion-irradiation at $77 \mathrm{~K}$.

The low energy recoil effects are tested by the comparison of MD simulation results with four mono-energetic recoils of $30,100,300,500 \mathrm{eV}$, and with the recoil spectrum simulation where low-energy recoils dominate. If possible healing of defects or recrystallization of amorphous pockets caused by low energy recoil is present, the result with the realistic recoil spectrum would show a slower amorphization rate, as reported for Si, for which the low-energy recoils between 3 and $15 \mathrm{eV}$ have been shown to slow down the amorphization by about a factor of 2 (Ref. 12). In the present simulations, however, the results with the realistic recoil spectrum are almost identical to the mono-energetic recoils, see Fig. 9. That indicates the amorphization of $\alpha$-quartz is not heavily dependent on the energy of the recoils. This conclusion is very well in line with the experimental observation [23] that the critical irradiation dose $1.92 \mathrm{eV} /$ atom for low-energy $(<\sim 100 \mathrm{keV}$ ) ion irradiation to make the quartz fully amorphous (as defined by RBS-C measures) is almost independent of ion species and energy, even though different kind of ions or same ions with different energies can generate quite different recoil spectra.

We also consider the possible network-collapsing effect of $\mathrm{Na}$ atoms implanted into the matrix (and possibly segregating). As our MD calculation does not explicitly include Na atoms, we use SRIM to calculate the concentration of $\mathrm{Na}$ at $45-90 \mathrm{~nm}$ (maximum damage) depth, and find it to be only $56 \mathrm{ppm}$. Its effect is thus negligible compared to the damages produced by the recoils. Also, an earlier study shows that $\mathrm{Na}$ segregates to the interface only at about $683 \mathrm{~K}$ during annealing [60].

To summarize this subsection, we found that the effects 1 and 2 do not contribute significantly to the amorphization processes of quartz. On the other hand, the different results on the degree of amorphization from the different measures clearly show that there is no unique measure of the degree of amorphization (effect 3). In the remainder of the paper we discuss these differences and how they help in understanding how the amorphization proceeds.

\section{B. Comparison of analysis models}

Comparison of the RBS-C yield, structure factor and Wigner-Seitz defect analysis can be seen in Fig. 10 . The critical doses for the amorphization of $\alpha$-quartz obtained by different methods is not identical, but ranges from 2.2 to $5.0 \mathrm{eV}$ /atom. Compared with other measurements, the RBS signal saturates to full amorphization at about 2.2 $\mathrm{eV}$ /atom, a smaller dose than that given by the other methods. This can be understood in terms of what the RBS channeling method actually measures: the fraction of atoms that are scattered back from defects in a crystal channel. As seen in the plot of $\langle 0001\rangle$ view of simulation cell shown by Fig. 11, the disorder is obviously increasing with the 


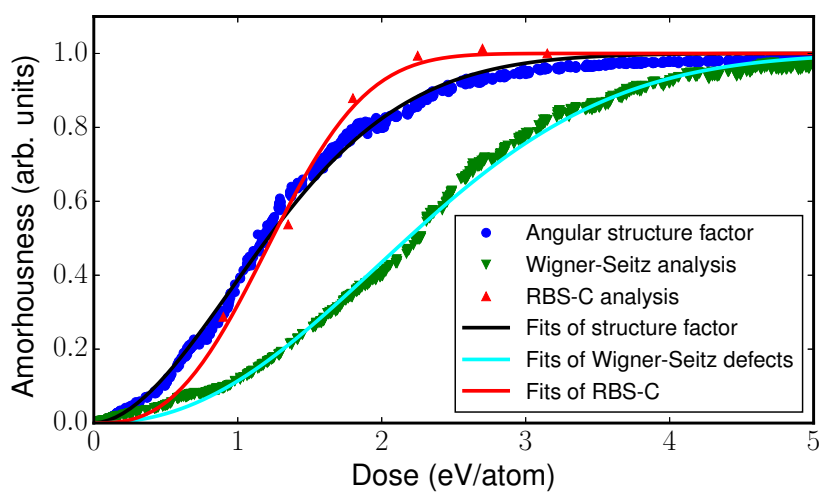

FIG. 10. Comparison of RBS-C, angular structure factor and Wigner-Seitz analysis and fits of the results by the nucleation and growth model.

irradiation dose. Fig. 11e with the $\langle 0001\rangle$ view of simulation cell at the dose $2.0 \mathrm{eV} /$ atom shows that the channels were still not fully "filled" even though the crystalline structure is heavily disordered, while almost no channel could be seen in the view with dose $2.5 \mathrm{eV}$ /atom (Fig. 11f). Thus, our results show that the RBS signals saturate at a dose lower than $2.5 \mathrm{eV} /$ atom.

The disorder in the local atomic neighborhood can keep increasing even after the maximum RBS yield is reached. This is shown by the angular structure factor and Wigner-Seitz defect analysis. The results show that even after the RBS signal has saturated, the angular distortions between bonds, the amount of Wigner-Seitz defects could still keep increasing to dose of 3.0 and $4.5 \mathrm{eV}$ /atom. The coordination defect, primitive ring analysis and atomic structure density analysis also showed that the structure continued to changes even after the RBS-C signal is saturated. The observation that the level of defects increases after the RBS maximum amorphization dose has been reached is in excellent agreement with the experimental observations, showing that the optical properties of ion irradiated quartz (related to the optically active defects) can keep changing up to a dose of $50 \mathrm{eV} /$ atom [6], even though the experimental RBS saturation has been reached already at the $2 \mathrm{eV} /$ atom level [23, 61]. The continued evolution of the structures makes the definition of what is, on atomic level, an amorphized quartz ambiguous.

As a final comment, we note that the fact that different analytical model fit the RBS, angular structure factor analysis and Wigner-Seitz defect number show independently that the analytical models available for in understanding the nature of amorphization provide a limited insight. As seen from the fits of RBS-C data (Fig. 3), angular structure factors (Fig. 4) and of the Wigner-Seitz defect number (Fig. 5), using the direct impact model to understand the nature of amorphization of quartz is not applicative, since it could not fit any one of the analysis data well. From the plot of the atomic structure (Fig. 11), we could find that the damage is clearly not, as assumed in the Gibbons damage overlap model, homogeneous, thus the Gibbons damage overlap model could not describe the process of amorphization of quartz, either. For the nucleation and growth model, even though it could fit all the analysis data well, the fitting parameters are obviously different. Thus it is concluded that on the atomic level, different measures of amorphization can give a quite different picture of how the disordering of a lattice proceeds.

\section{CONCLUSIONS}

We have simulated the ion beam amorphization of quartz by means of classical molecular dynamics simulations, and analyzed the resulting structures with RBS-C simulations as well as angular structure factor, Wigner-Seitz defects, radial distribution, coordination, ring structure and density analysis. The RBS-C results were shown to be in good agreement with experiments, considering that no fitting parameters were used for the comparison. On the other hand, the different analysis methods gave clearly different evolution behavior as a function of dose. This was shown by the angular structure factor, the fraction of Wigner-Seitz defects, coordination defects, ring structure and density of structure still evolving after the backscattering signal saturated. The results show on the atomic level that different measures of amorphization can give a quite different picture of how the disordering of a lattice proceeds, in particular that a saturated RBS channeling signal does not necessarily mean that the microstructure of a sample has stopped evolving, and the continued evolution of structures makes the definition of amorphized quartz ambiguous. 


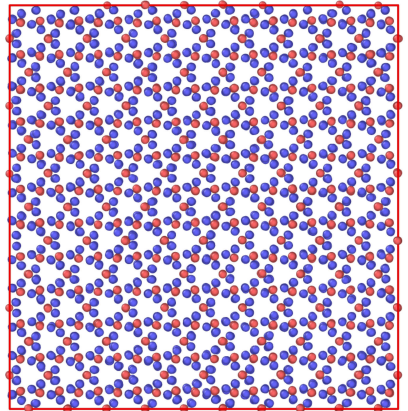

(a)

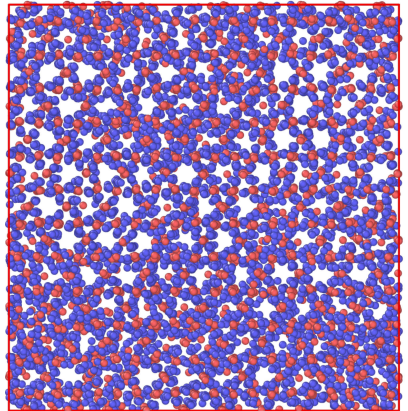

(c)

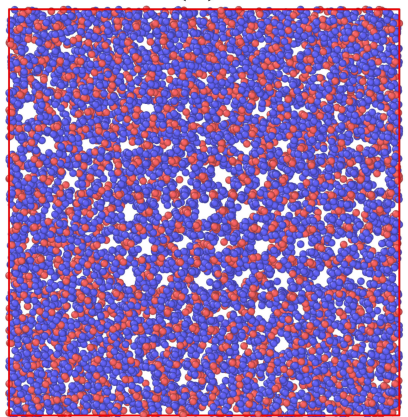

(e)

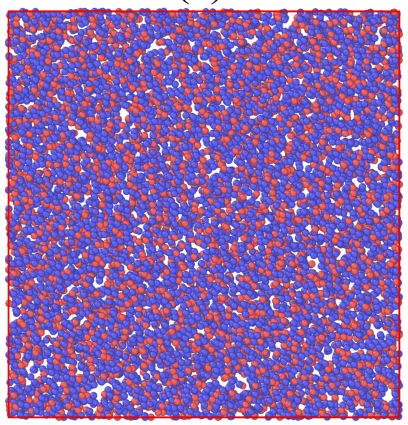

(g)

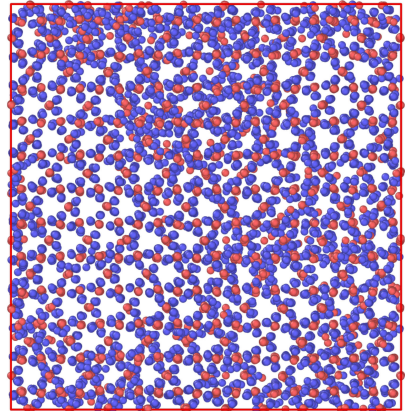

(b)

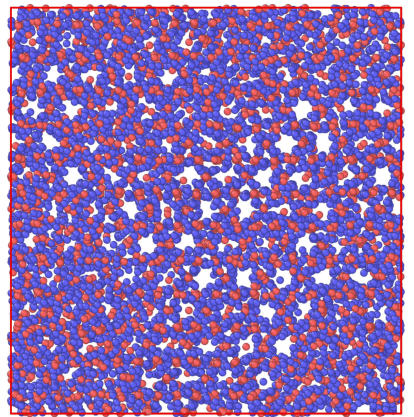

(d)

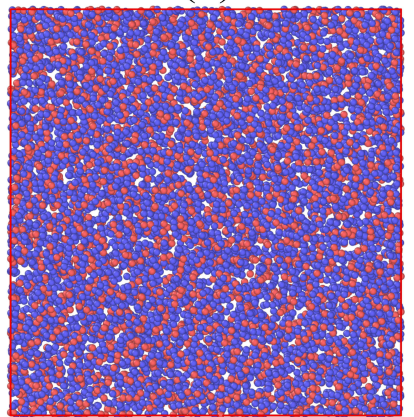

(f)

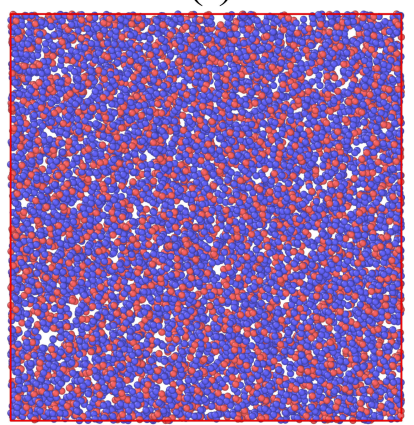

(h)

FIG. 11. $\langle 0001\rangle$ view of MD simulation cells with irradiation dose (a) $0.0 \mathrm{eV} /$ atom, (b) $0.5 \mathrm{eV} /$ atom, (c) $1.0 \mathrm{eV} / \mathrm{atom},(\mathrm{d}) 1.5$ $\mathrm{eV} /$ atom, (e) $2.0 \mathrm{eV} /$ atom, (f) $2.5 \mathrm{eV} /$ atom, (g) $3.0 \mathrm{eV} /$ atom, (h) $5.0 \mathrm{eV} /$ atom. The size of cells is $\sim 5 \times 5 \times 5 \mathrm{~nm}^{3}, \mathrm{the} \mathrm{red}^{\circ}$ balls denote $\mathrm{Si}$ atoms and blue balls denote $\mathrm{O}$ atoms.

\section{ACKOWLEDGEMENTS}

This work was performed within the Finnish Centre of Excellence in Computational Molecular Science (CMS), financed by The Academy of Finland and the University of Helsinki. Grants of computer time from the Center for Scientific Computing in Espoo, Finland, are gratefully acknowledged. We also acknowledge grants of computer capacity from the Finnish Grid and Cloud Infrastructure (persistent identifier urn:nbn:fi:research-infras-2016072533) 
and the supports of National Natural Science Foundation of China (Grant Nos. 11505085, 11505084). The authors F. Djurabekova and K. Nordlund acknowledge the funding received from the European Union's Horizon 2020 research and innovation programme under grant agreement No. 688072.

[1] J. Narayan, D. Fath, O. S. Oen, and O. W. Holland, J. Vac. Sci. Technol. A 2, 1303 (1984).

[2] M. O. Ruault, J. Chaumont, J. M. Penisson, and A. Bourret, Phil. Mag. A 50, 667 (1984).

[3] D. N. Seidman, R. S. Averback, P. R. Okamoto, and A. C. Baily, Phys. Rev. Lett. 58, 900 (1987).

[4] T. Motooka and O. W. Holland, Appl. Phys. Lett. 58, 2360 (1991).

[5] K. Nordlund, J. Keinonen, E. Rauhala, and T. Ahlgren, Phys. Rev. B 52, 15170 (1995).

[6] L. Douillard and J. Duraud, Nucl. Instr. Meth. Phys. Res. B 107, 212 (1996).

[7] E. Chason, S. T. Picraux, M. Poate, J. O. Borland, M. I. Current, T. Diaz de la Rubia, D. J. Eaglesham, O. W. Holland, M. E. Law, C. W. Magee, J. W. Mayer, J. Melngailis, and A. F. Tasch, J. Appl. Phys 81, 6513 (1997).

[8] R. S. Averback and T. Diaz de la Rubia, in Solid State Physics, Vol. 51, edited by H. Ehrenfest and F. Spaepen (Academic Press, New York, 1998) pp. 281-402.

[9] K. Gärtner and B. Weber, Nucl. Instr. Meth. Phys. Res. B 180, 274 (2001).

[10] F. Gao, W. J. Weber, and R. Devanathan, Nucl. Instr. Meth. Phys. Res. B 140, 176 (2001).

[11] C. J. Glover, K. M. Yu, M. C. Ridgway, and G. J. Foran, Jap. J. Appl. Phys. 38, 548 (1999).

[12] J. Nord, K. Nordlund, and J. Keinonen, Phys. Rev. B 65, 165329 (2002).

[13] E. Wendler, A. Kamarou, E. Alves, K. Gärtner, and W. Wesch, Nucl. Instr. Meth. Phys. Res. B 206, 1028 (2003).

[14] K. Trachenko, J. M. Pruneda, E. Artacho, and M. T. Dove, Phys. Rev. B 71, 184104 (2005).

[15] B. Wang, N. M. A. Krishnan, Y. Yu, M. Wang, Y. L. Pape, G. Sant, and M. Bauchy, J. Non-Cryst. Solids 463, 25 (2017).

[16] J. Nord, K. Nordlund, and J. Keinonen, Nucl. Instr. Meth. Phys. Res. B 193, 294 (2002).

[17] S. C. Glotzer, J. Non-Cryst. Solids 274, 342 (2000).

[18] V. S. Varichenko, A. M. Zaitsev, J. Lindner, R. Domres, N. M. Penina, D. Erchak, A. Chelyadinskii, and V. Martinovitsh, Nucl. Instr. Meth. Phys. Res. B 94, 240 (1994).

[19] M. P. Allen and D. J. Tildesley, Computer Simulation of Liquids (Oxford University Press, Oxford, England, 1989).

[20] J. Nord, K. Nordlund, and J. Keinonen, Phys. Rev. B 68, 184104 (2003).

[21] J. W. Mayer and S. S. Lau, Electronic Materials Science For Integrated Circuits in Si and GaAs (MacMillan, New York, 1990).

[22] L. Khriachtchev, ed., Silicon nanophotonics: Basic Principles, Present Status and Perspectives (World Scientific, Singapore, 2008).

[23] F. Harbsmeier and W. Bolse, J. Appl. Phys. 83, 4049 (1998).

[24] H. Matzke, phys. stat. sol. (b) 18, 285 (1966).

[25] H. Fischer, G. Götz, and H. Karge, Phys. Stat. Sol. 76, 249-256 (1983).

[26] H. Fischer, G. Götz, and H. Karge, Phys. Stat. Sol. 76, 493 (1983).

[27] F. Roccaforte, M. J. Gustafsson, W. Bolse, J. Keinonen, and K. P. Lieb, Nucl. Instr. Meth. Phys. Res. B 166-167, 148 (2000).

[28] K. P. Lieb and J. Keinonen, Contemporary Physics 47, 305 (2006).

[29] F. Mota, M.-J. Caturla, J. M. Perlado, A. Ibarra, M. León, and J. Mollá, J. Nucl. Mater. 367-370, 344 (2007).

[30] F. Mota, M.-J. Caturla, J. M. Perlado, , J. Molla, and A. Ibarra, J. Nucl. Mater. 386-388, 75 (2009).

[31] N. M. A. Krishnan, B. Wang, Y. L. Pape, G. Sant, and M. Bauchy, J. Chem. Phys. 146, 144 (2017).

[32] S. Plimpton, J. Comput. Phys. 117, 1 (1995).

[33] J. Q. Broughton, C. A. Meli, P. Vashishta, and R. K. Kalia, Phys. Rev. B 56, 611 (1997).

[34] K. Nordlund, Comput. Mater. Sci. 3, 448 (1995).

[35] A presentation of the MDRANGE computer code is available on the World Wide Web in http://beam.acclab.helsinki.fi/ knordlun/mdh/mdh_program.html.

[36] Y. Zhang, M. Ishimaru, J. Jagielski, W. Zhang, Z. Zhu, L. V. Saraf, W. Jiang, L. Thome, and W. J. Weber, J. Phys. D: Appl. Phys. 43, 085303 (2010).

[37] L. Zhang, W. Jiang, A. Dissanayake, J. Peng, W. Ai, J. Zhang, Z. Zhu, T. Wang, and V. Shutthanandan, J. Appl. Phys. 119, 245704 (2016), http://dx.doi.org/10.1063/1.4954691.

[38] S. Zhang, K. Nordlund, F. Djurabekova, Y. Zhang, G. Velisa, and T. S. Wang, Phys. Rev. E 94, 043319 (2016).

[39] S. Zhang, K. Nordlund, F. Djurabekova, F. Granberg, Y. Zhang, and T. S. Wang, Mater. Res. Lett. 5, 433 (2017).

[40] J. F. Ziegler, SRIM-2013 software package, available online at http://www.srim.org.

[41] H. Zhu, R. S. Averback, and M. Nastasi, Phil. Mag. A 71, 735 (1995).

[42] K. Nordlund and R. S. Averback, Phys. Rev. B 56, 2421 (1997).

[43] F. Djurabekova and K. Nordlund, Phys. Rev. B 77, 115325 (2008), also selected to Virtual Journal of Nanoscale Science \& Technology Vol. 17 Issue 13 (2008).

[44] A. Stukowski, Modell. and Simul. in Mat. Sci. and Eng. 18, 015012 (2010).

[45] C. Jesurum, V. Pulim, and L. W. Hobbs, Nucl. Instrum. Meth. B 141, 25 (1998).

[46] C. E. Jesurum, V. Pulim, and L. W. Hobbs, J. Nucl. Mater. 253, 87 (1998). 
[47] X. Yuan, V. Pulim, and L. W. Hobbs, J. Nucl. Mater. 289, 71 (2001).

[48] L. W. Hobbs, Nucl. Instr. Meth. Phys. Res. B 91, 30 (1994).

[49] H. Foxhall, K. Travis, L. Hobbs, S. Rich, and S. Owens, Philosophical Magazine 93, 328 (2013).

[50] S. L. Roux and P. Jund, Comp. Mat. Sci. 49, 70 (2010).

[51] K. Fang, J. Zou, E. Yoshida, T. Wang, and J. Kasagi, EPL (Europhysics Letters) 109, 22002 (2015).

[52] F. Kai-Hong, Z. Jian-Xin, L. Dong-Dong, Z. Jiang-Tao, and W. Tie-Shan, Chinese physics C 39, 084001 (2015).

[53] K. Nordlund, F. Djurabekova, and G. Hobler, Phys. Rev. B 94, 214109 (2016).

[54] J. R. Dennis and E. B. Hale, J. Appl. Phys. 49, 1119 (1978).

[55] J. F. Gibbons, Proceedings of the IEEE 60, 1062 (1972).

[56] S. U. Campisano, S. Coffa, V. Rainieri, F. Priolo, and E. Rimini, Nucl. Instr. Meth. Phys. Res. B 80/81, 514 (1993).

[57] L. Pelaz, L. A. Marqués, and J. Barbolla, J. Applied Physics 96, 5947 (2004).

[58] M. Wittels and F. A. Sherrill, Phys Rev 93, 1117 (1954).

[59] E. Holmström, B. Haberl, O. Pakarinen, K. Nordlund, F. Djurabekova, R. Arenal, J. S. Williams, J. E. Bradby, T. C. Petersen, and A. C. Y. Liu, J. Non-Cryst. Solids 438, 26 (2016).

[60] P. Haussalo, J. Keinonen, K. P. Lieb, W. Bolse, and C. Illgner, Nucl. Instr. Meth. Phys. Res. B 120, 266 (1996).

[61] F. Harbsmeier and W. Bolse, Materials Science Forum 248-249, 279 (1997). 